\title{
Nanocrystalline $\alpha$-Fe Layer Examined by Mössbauer Spectrometry
}

\author{
B. David ${ }^{a, *}$, O. Schneeweiss ${ }^{a}$, N. Pizúrová ${ }^{a}$, A. ReK ${ }^{b}$, V. Kudrle ${ }^{c}$, O. JAŠEK $^{c, d}$ \\ ${ }^{a}$ CEITEC IPM, Institute of Physics of Materials, ASCR, v.v.i., Žižkova 22, CZ 61662 Brno, Czech Republic \\ ${ }^{b}$ Institute of Scientific Instruments, ASCR, v.v.i., Královopolská 147, CZ 61264 Brno, Czech Republic \\ ${ }^{c}$ DPE, Faculty of Science, Masaryk University, Kotlářská 2, CZ 61137 Brno, Czech Republic \\ ${ }^{d}$ CEITEC MU, Masaryk University, Kamenice 753/5, CZ 62500 Brno, Czech Republic \\ A few micrometers thick nanocrystalline $\alpha$-Fe layer with the mean crystallite size $d_{X R D}=14 \mathrm{~nm}$ was deposited \\ in low-pressure microwave plasma, using $\mathrm{Fe}(\mathrm{CO})_{5}$ vapour. Its nanocrystalline character was proved on its surface \\ under SEM (surface was formed of deposited nanoparticles) and in its volume using TEM (deposited nanoparticles \\ were stacked up, creating columns). No significant iron oxide phases were observed in the transmission ${ }^{57} \mathrm{Fe}$ \\ Mössbauer spectrum measured at $5 \mathrm{~K}$ nor in the surface-sensitive ${ }^{57} \mathrm{Fe}$ conversion electron Mössbauer spectrum \\ measured at $293 \mathrm{~K}$.
}

DOI: 10.12693 /APhysPolA.126.94

PACS: 52.75.Hn; 73.63.Bd; 76.80.Pj

\section{Introduction}

Microwave-plasma-assisted chemical vapour deposition (MPCVD) is a well-established method for the synthesis of nanocrystlline films [1, 2]. As a fundamental material, the nanograined $\alpha$-Fe layer is a desirable topic for investigation. Nevertheless, the studies of nanocrystalline $\alpha$-Fe layers deposited by a MPCVD method are rare.

In this paper we present results obtained by the study of a few micrometers thick nanocrystalline $\alpha$-Fe layer deposited in low-pressure microwave plasma.

\section{Experimental methods}

Our experimental setup was straightforward and similar to the one used in our previous work [3]. Operating conditions were as follows: gas pressure of $2 \mathrm{kPa}$, microwave power of $250 \mathrm{~W}$, Ar flow of $280 \mathrm{sccm}$, and $\mathrm{Fe}(\mathrm{CO})_{5}$ flow of $1.75 \mathrm{~g} / \mathrm{min}$. The $\mathrm{Fe}(\mathrm{CO})_{5}$ injection point (quartz tube with $6 \mathrm{~mm}$ inner diameter) was situated $15 \mathrm{~cm}$ downstream from the launcher (surfaguide). Deposition time was 60 seconds. $\alpha$-Fe thin films were deposited on the reactor inner wall of $45 \mathrm{~mm}$ quartz tube close to the launcher and also on the inner wall of the most upper part of injection tube.

X-ray diffraction (XRD) pattern was measured on a PANalytical X'Pert Pro MPD device and pattern fitting was done using X'Pert High Score Plus 2.0a and ICSD database and it yielded mean crystallite size $d_{X R D}$. Scanning electron microscopy (SEM) was done on a JEOL JSM 6700F. Transmission electron microscopy (TEM) was performed on a Philips microscope CM12. The foil for TEM observations was prepared by applying ion machining to the original sample. Transmission ${ }^{57} \mathrm{Fe}$ Mössbauer spectrum (TMS) was obtained at standard transmission geometry with ${ }^{57} \mathrm{Co}$ in $\mathrm{Rh}$ matrix source. As a result of the fitting procedure, performed with CONFIT

* corresponding author; e-mail: david@ipm.cz software package, we obtained relative spectrum area $A$ for a given component and spectral component parameters: hyperfine magnetic induction $B_{H F}$, width of hyperfine Gaussian distribution $\Delta B_{H F}$, quadrupole shift $\varepsilon_{Q}$, quadrupole splitting $\Delta E_{Q}$, isomer shift $\delta$ (against $\alpha-\mathrm{Fe}$ ), and line intensity $I$. A CCS-800 Mössbauer closed-cycle refrigerator system from Janis was used for low temperature measurement. Conversion electron Mössbauer spectrum (CEMS) was measured at room temperature using a gas flow (90 vol.\% He, 10 vol. $\% \mathrm{CH}_{4}$ ) electron detector.

\section{Results}

Under a conventional light microscope the pieces of the deposited layer exhibited generally metallic shine, but on some places of the surface also macroscopic reddishbrown areas (about $20 \%$ of the whole surface) were observed, indicating possible oxidation of the surface.

The XRD pattern of the as-synthesized pieces of the layer exhibited only $\alpha$-Fe diffraction lines (ICSD \#53451, $\left.R_{w p}=5.41, \mathrm{GOF}=24.7\right)$ with the mean crystallite size $d_{X R D}=14 \mathrm{~nm}$. No other significant peaks were observed.

The morphology of the broken piece of the layer was examined under SEM (Fig. 1a) and it revealed that the surface of the layer consisted of deposited nanoparticles with the diameter of about $20 \mathrm{~nm}$ (Fig. 1b). The shape of the fracture surface of the layer exhibited such a structure as if the volume of the layer contained columns, which could be formed of nanoparticles. TEM confirmed this assumption (Fig. 2): the grains had the tendency to be stacked up in columns oriented nearly perpendicularly to the surface.

To supress superparamagnetism in small particles /grains and so being able to observe the spectral components (esp. those with $B_{H F}>\alpha-\mathrm{Fe}$ ) belonging to possible iron oxide compounds with magnetic ordering, the TMS for the sample was measured at $5 \mathrm{~K}$ (Fig. 3). However, it was not significantly different form the roomtemperature TMS. The low-temperature TMS was fitted with $\alpha$-Fe sextet $\left(B_{H F}=33.9 \mathrm{~T}, 2 \varepsilon_{Q}=0.00 \mathrm{~mm} / \mathrm{s}\right.$, 
$\delta=0.14 \mathrm{~mm} / \mathrm{s}, A=0.78, I_{2} / I_{1}=0.65$, full line), Gaussian distribution $\left(B_{H F}=31.9 \mathrm{~T}, \Delta B_{H F}=13.1 \mathrm{~T}\right.$, $2 \varepsilon_{Q}=0.00 \mathrm{~mm} / \mathrm{s}, \delta=0.20 \mathrm{~mm} / \mathrm{s}, A=0.19$, dashed line), and a doublet $\left(\Delta E_{Q}=0.36 \mathrm{~mm} / \mathrm{s}, \delta=0.26 \mathrm{~mm} / \mathrm{s}\right.$, $A=0.03$, full line). The spectrum fit is shown with bold full line. The distribution was assigned to Fe atoms at the interfaces of the grains [4]. No components characteristic for iron oxides were found [5].
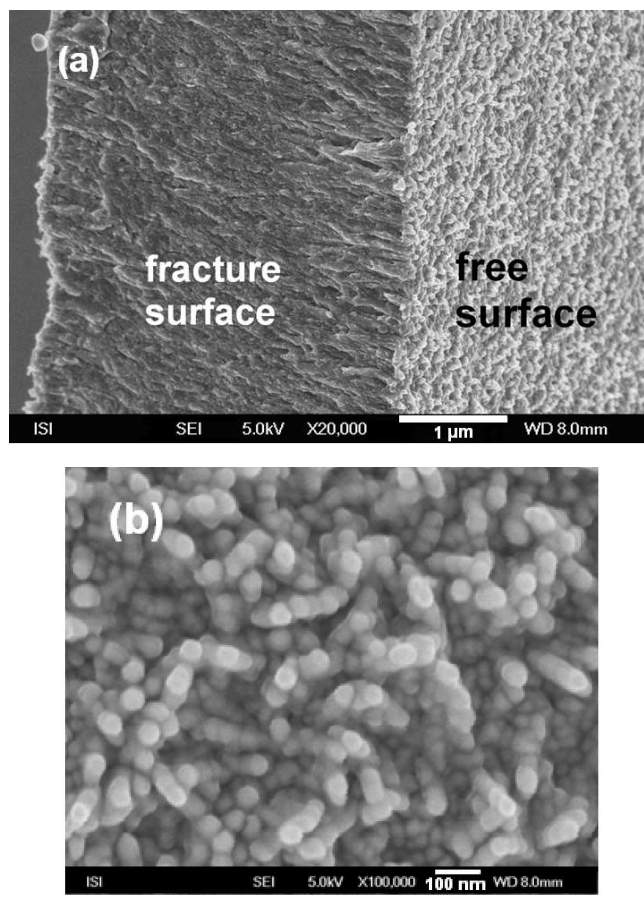

Fig. 1. SEM images for (a) the edge of a piece of $\alpha$-Fe layer and (b) its free surface viewed from above.

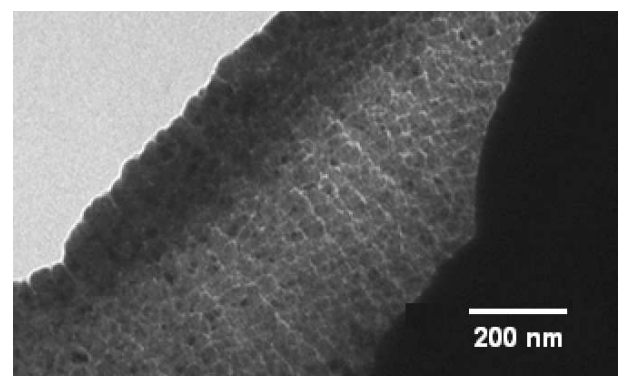

Fig. 2. TEM image for the thin foil prepared from a piece of $\alpha$-Fe layer.

CEMS served to investigate the surface layer with the thickness of about $300 \mathrm{~nm}$ (the approximate escape depth of conversion electrons). The CEMS spectrum was fitted with $\alpha$-Fe sextet $\left(B_{H F}=32.9 \mathrm{~T}, 2 \varepsilon_{Q}=0.00 \mathrm{~mm} / \mathrm{s}\right.$, $\delta=0.02 \mathrm{~mm} / \mathrm{s}, A=0.85, I_{2} / I_{1}=0.79$, full line), Gaussian distribution $\left(B_{H F}=31.3 \mathrm{~T}, \Delta B_{H F}=28.5 \mathrm{~T}\right.$, $2 \varepsilon_{Q}=0.00 \mathrm{~mm} / \mathrm{s}, \delta=0.00 \mathrm{~mm} / \mathrm{s}, A=0.14$, dashed line), and a singlet ( $\delta=0.09 \mathrm{~mm} / \mathrm{s}, A=0.01$, full line).
The spectrum fit is shown with bold full line. No clearly assignable iron oxide components were observed.

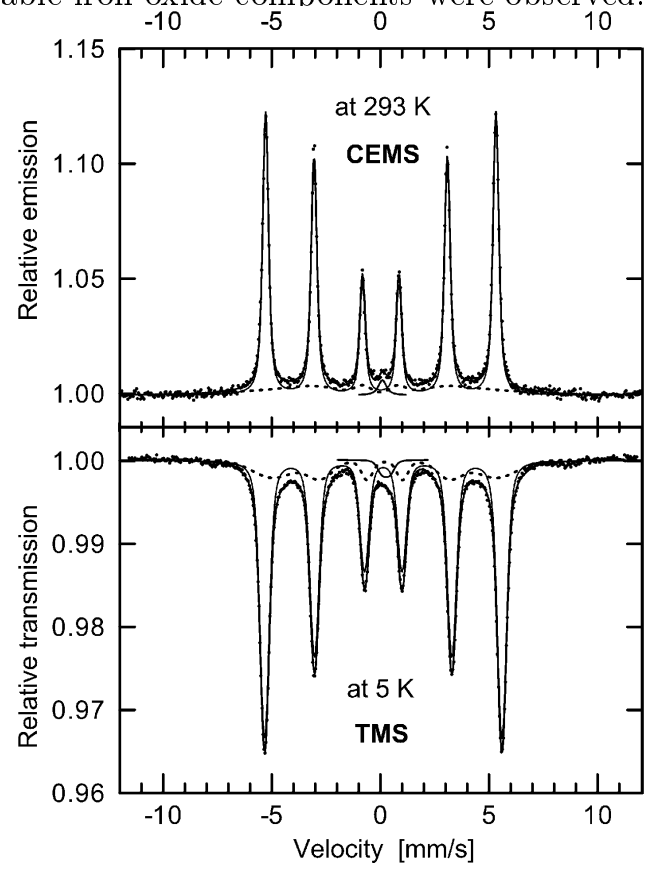

Fig. 3. MS spectra for a few pieces of $\alpha$-Fe layer.

\section{Conclusions}

Although the layers had macroscopic reddish-brown areas on their surfaces, which might be iron oxides, no iron oxide phases in significant amounts were identified in TMS spectrum nor CEMS spectrum. Hence, the reddishbrown areas on the surfaces could be some byproducts of the decomposition of $\mathrm{Fe}(\mathrm{CO})_{5}[6]$, exhibiting themselves in the middle part of the Mössbauer spectra.

\section{Acknowledgments}

This work was realized in CEITEC - Central European Institute of Technology with research infrastructure supported by the project CZ.1.05/1.1.00/02.0068 financed from European Regional Development Fund and in the R\&D centre for low-cost plasma and nanotechnology surface modifications CZ.1.05/2.1.00/03.0086 funded by European Regional Development Fund.

\section{References}

[1] K.L. Choy, Prog. Mater. Sci. 48, 57 (2003).

[2] S.C. Tjong, H. Chen, Mater. Sci. Eng. $R$ 45, 1 (2004).

[3] B. David, N. Pizúrová, O. Schneeweiss, E. Šantavá, O. Jašek, V. Kudrle, J. Phys.: Conf. Series 303, 012090 (2011).

[4] R.J. Pollard, J. Chadwick, Hyperfine Interactions 94, 2245 (1994).

[5] R.M. Cornell, U. Schwertmann, The Iron Oxides Wiley-VCH, 2003.

[6] R. Cireasa, R. Alexandrescu, I. Voicu, I. Morjan, G. Pugna, Surf. Coat. Tech. 80, 229 (1996). 